\title{
Numerical Modeling of Surface Roughness Effects on the Natural Frequency of a Silicon Cantilever
}

\author{
Jean Marriz Manzano${ }^{1}$, Magdaleno Vasquez $^{2}$, Marc Rosales ${ }^{1}$, Maria Theresa De Leon ${ }^{1}$ \\ ${ }^{1}$ Electrical and Electronics Institute/University of the Philippines Diliman \\ Quezon City, Philippines \\ jean.manzano@eee.upd.edu.ph; marc.rosales@eee.upd.edu.ph; theresa.de.leon@eee.upd.edu.ph; \\ ${ }^{2}$ Department of Mining, Metallurgical, and Materials Engineering/University of the Philippines Diliman \\ Quezon City, Philippines \\ mrvasquez@up.edu.ph;
}

\begin{abstract}
This paper presents a numerical model showing the effects of surface roughness on the resonant frequency of a cantilever beam. The model developed incorporates non-uniform cross-sections to represent surface roughness. Results show that as roughness increases, resonant frequency increases. The model presented here also has good agreement with FEM simulations.
\end{abstract}

Keywords: Deep reactive ion etching, Finite Element Method, Microelectromechanical Systems, Surface roughness

\section{Introduction}

Most MEMS devices use a silicon substrate because of its good mechanical properties. Silicon is also most commonly used in MEMS to form cantilever beams. During fabrication, certain processes cause surface irregularities that influence not only the surface properties of a device but also its reliability and performance [1,4,8,9]. For cantilevers that are used for energy harvesting, this may lead to harvesting inefficiency as its operating frequency is highly dependent on its geometric shape. Etching of silicon using deep reactive ion etching (DRIE) at large etch depths results in rougher surfaces [2] due to an increased response in process pressure, amount of coil power and increased helium backside cooling readings. Note that the resulting surface roughness changes the moment of inertia per cross-section of the cantilever. This paper presents a numerical model that can be used to calculate the natural frequency of a silicon cantilever beam, which includes the effects of the cantilever's non-uniform cross-sectional heights caused by surface roughness.

\section{Numerical Modeling of Surface Roughness}

In approximating the natural frequency of a fixed-free cantilever beam that is subjected to free vibrations, available mathematical models assume that the cross section of the beam is uniform [5-7]. Hence, the beam's moment of inertia is considered uniform all throughout its entire length. In this paper, the numerical model incorporates both the device properties and surface roughness to predict the resonant frequency of a cantilever. To do this, measurement and characterization of the surface roughness of sample structures were performed first. These samples have undergone a standard DRIE MEMS fabrication process with an etch rate of $750 \mathrm{~nm} /$ cycle using $\mathrm{C}_{4} \mathrm{~F}_{8}, \mathrm{SF}_{6}$, and $\mathrm{O}_{2}$ gases.

The root mean square roughness parameter, $S_{q}$ was used for characterizing the surface roughness [3], and is obtained by squaring each height value and taking the square root of the mean. Four samples were measured at the same location per region of a 6-inch wafer and statistical information was acquired using a high-resolution 3D laser microscope. Roughness was characterized and analyzed using the microscope's software data analysis tool. Areal method was used to acquire roughness of the surface which region of measurement was specified to be $640 \times 640 \mu \mathrm{m}$. Sample images of the surface roughness data are shown in figure 1.

Etch depth of the wafer was measured to fairly quantify surface roughness. Thickness measurement results for a DRIE 6-inch wafer is shown in figure 2. It is found that the center region is thicker compared to other regions of the wafer. The average thickness of the center region is approximately $120 \mu \mathrm{m}$ while the rest of the wafer's thickness ranges from $90-100$ $\mu \mathrm{m}$. Acquired roughness using parameter, $S_{q}$ shows that the center region of the wafer tends to have an average of 
approximately $2.5 \mu \mathrm{m}$ while the left and right regions have more variations compared to the bottom, top and the center regions. Results of the measurement after characterization is shown in figure 3.

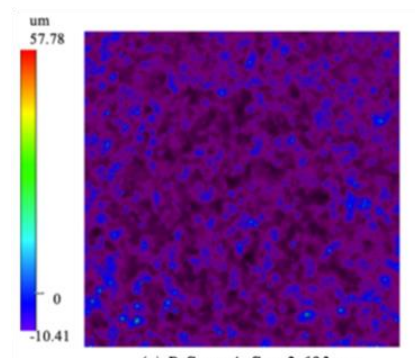

(a) B-Samp4: $S_{q}=2.683 \mu m$

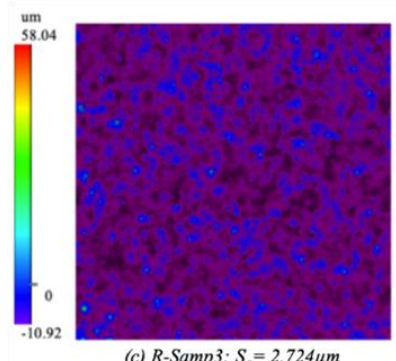

(c) R-Samp3: $S_{q}=2.724 \mu \mathrm{m}$
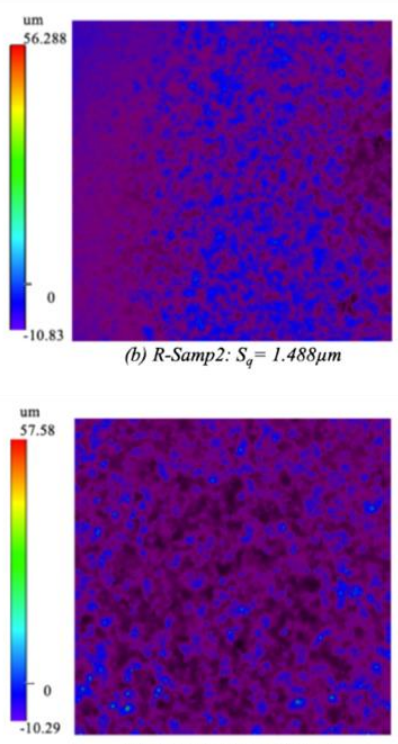

(d) T-Samp1: $S_{q}=3.138 \mu m$

Fig. 1: Selected samples of roughness height images (1024x1024 px, 20x mag) using high resolution 3D laser microscope at $100 \mu \mathrm{m}$ thickness of a silicon wafer after 600 cycles of DRIE.

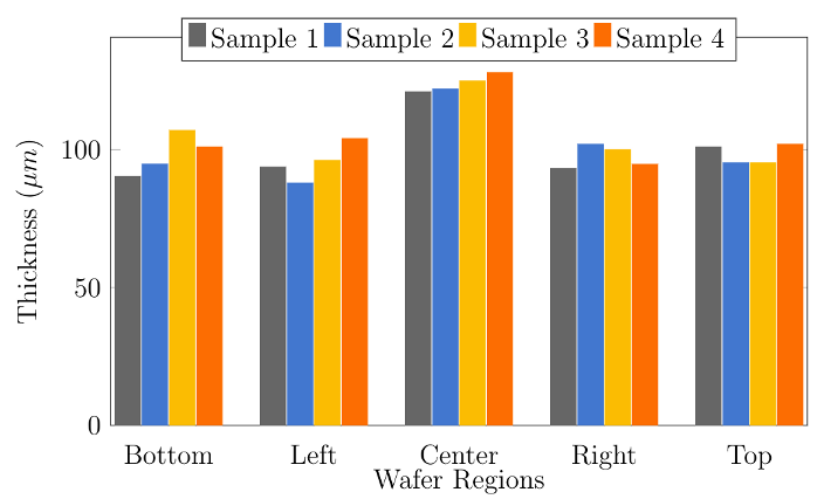

Fig. 2: Thickness variation across a 6-inch silicon wafer after performing DRIE. The center region of the wafer has the highest thickness value indicating least silicon etching rate at this region.

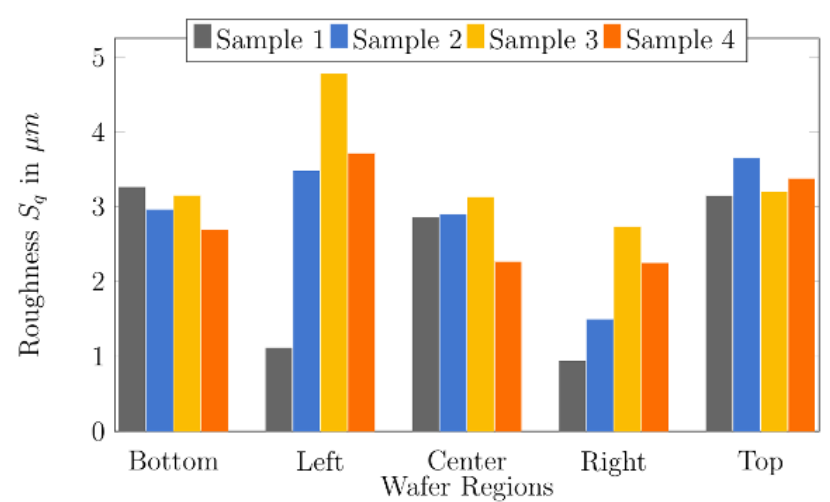

Fig. 3: Variation in surface roughness, $S_{q}$, across a 6inch silicon wafer after performing DRIE. It can be seen that roughness, $S_{q}$, can range from $1-4.7 \mu \mathrm{m}$ across the wafer.

Euler-Bernoulli's natural frequency equation of a cantilever beam shown in equation 1 calculates moment of inertia with the assumption that the beam's cross-section is uniform throughout its entire length. Illustrated in figure 4, is the rectangular cross-section's second moment of area on either axis with respect to its centroid given by a general form in equation 2.

$$
\omega_{n}=\frac{1}{2 \pi}\left(\frac{\beta_{n}}{L}\right)^{2} \sqrt{\frac{E I}{\rho A}}
$$




$$
I=\int d I=\int_{0}^{M} r^{2} d m
$$

The differential element $d m$ of the moment of inertia $d I$ is defined with respect to a specific rotational axis. In figure $4 \mathrm{a}$, the beam is bending about the $\mathrm{z}$-axis with respect to its central axis. Figure $4 \mathrm{~b}$, calculates $I z$ across the length $b$ and width $h$ from the centroid and integrates all the mass elements of the cross-section. This results in a second moment of area as described in equation 2. Breaking this area integral and evaluating it results to an area moment of inertia for a rectangular beam with cross-section used in equation 1.

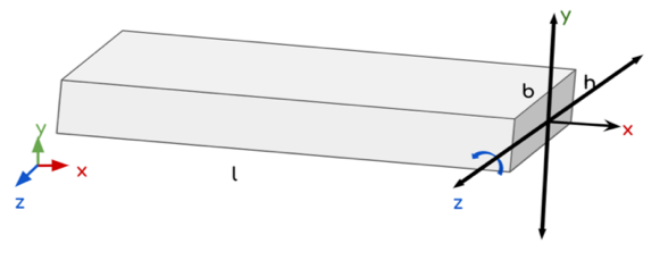

(a) Beam rotating at $\mathrm{z}$-axis

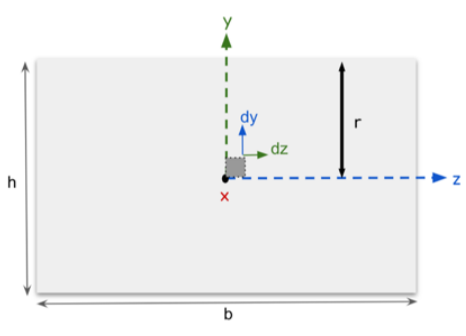

(b) Cross-section of a beam

Fig. 4: Illustration of moment of inertia of a cantilever beam with uniform thickness.

The numerical model in this paper, implemented in MATLAB, modifies equation 2 by using the analysis of surface roughness as varying heights. The length of the illustrated beam in figure $5 \mathrm{a}$ was subdivided equally as one slice of the crosssection, $C_{n}$ as shown in figure $5 \mathrm{~b}$. The cross-section was decomposed into uniform $d x$ segments that runs along the width of the beam as illustrated in figure 5c. Note that every $d y$ segment is the value of the total thickness per $d x$ segment. The total thickness differs depending on the (1) statistical information acquired from the microscope and (2) the calculated moment of inertia.

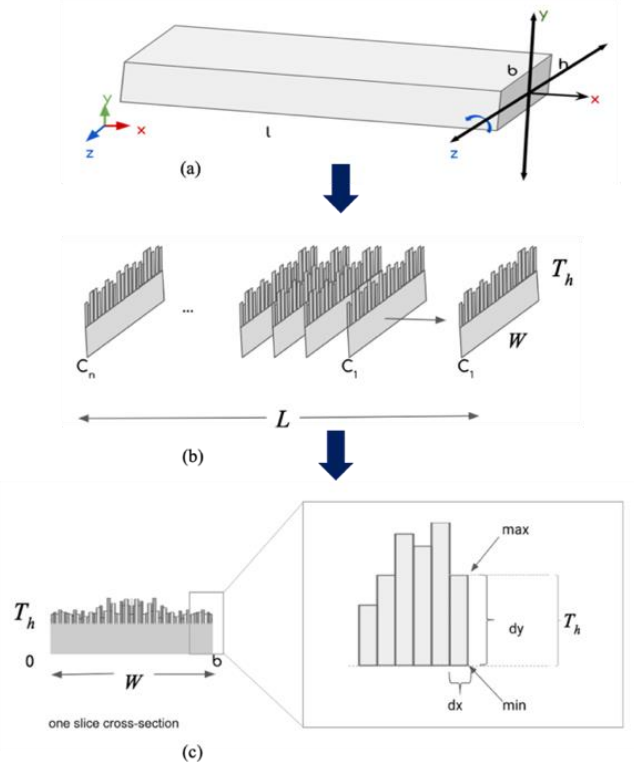

Fig. 5: Illustration of a (a) fixed-free cantilever beam with surface roughness represented by (b) varying cross-sectional heights. In the numerical model developed, the average moment of inertia and average cross-sectional in (c) are calculated using equation 3 and 4 , respectively. The cantilever's resonant frequency is then determined using equation 5. 
The pertinent equations used in the model to represent roughness as variations in the cross-sectional heights of the cantilever are also provided. Expressing the average moment of inertia that describes the beam with non-uniform thickness gives:

$$
I_{\text {ave }}=\frac{1}{L} \int_{0}^{L} \int_{0}^{b}\left(\int_{h \min }^{h m a x} y^{2} d A\right) d w d L
$$

and for the consistency of the natural frequency equation in 1, the area is expressed as:

$$
A_{\text {ave }}=\frac{1}{L} \int_{0}^{L} \int_{0}^{b} f(w) d w d L
$$

The newly computed $I_{\text {ave }}$ and $A_{\text {ave }}$ replaces the area moment of inertia and the beam's cross-sectional area of a cantilever beam natural frequency as shown in equation 5 .

$$
\omega_{n}^{\prime}=\frac{1}{2 \pi}\left(\frac{\beta_{n}}{L}\right)^{2} \sqrt{\frac{E * I_{\text {ave }}}{\rho * A_{\text {ave }}}}
$$

To test the functionality of the modified equation, acquired surface roughness was integrated to the moment of inertia. This was done by assuming a cantilever beam's total thickness height to be divided into two parts namely, $T_{l}$ and $T_{2}$ as presented in figure 6.

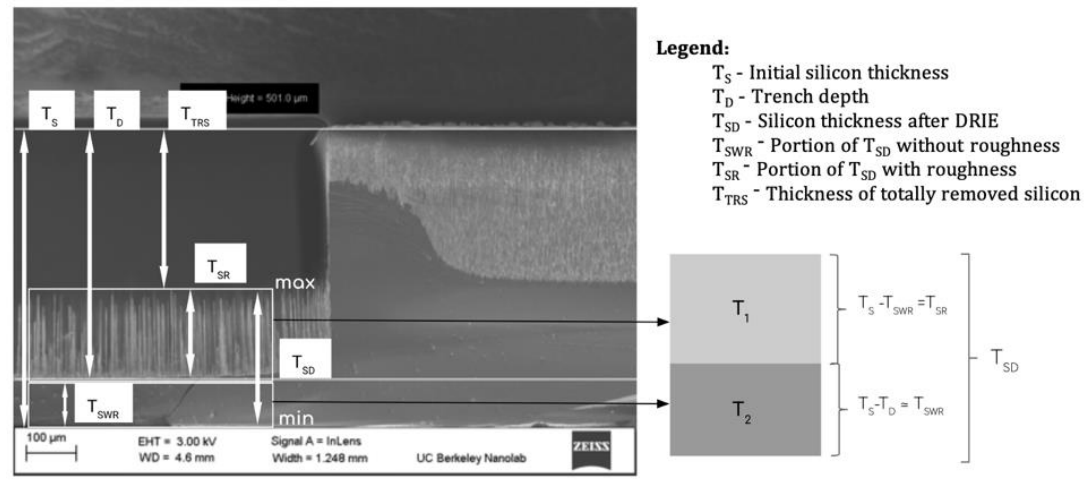

Fig. 6: Defining thickness on an SEM image of a silicon structure after deep reactive ion etching (DRIE).

Thickness $T_{1}$ represents the portion of silicon with surface roughness and varies depending on the maximum height value of the captured image. Meanwhile, thickness $T_{2}$ represents the silicon beam without roughness, with a value that is approximately $100 \mu \mathrm{m}$. Then, the material properties of the silicon beam that was acquired from COMSOL's material library was set as well as its boundary conditions for a fixed-free cantilever beam. Finally, the natural frequency of a cantilever beam incorporated with roughness was computed in MATLAB using equation 5.

\section{Comparison of Numerical Model with FEM Simulations}

To verify the numerical model developed, multiphysics model of a cantilever beam with surface roughness was simulated through FEM in COMSOL. Eigenfrequency and frequency domain analysis were performed to determine the natural frequency of the cantilever with surface roughness. A comparison was made between the analytical model 
and FEM simulations. Figure 7 shows the comparison between the numerical model and FEM simulations for five $100 \mu \mathrm{m}-$ thick cantilever beams with roughness characteristics as shown in figure 1.

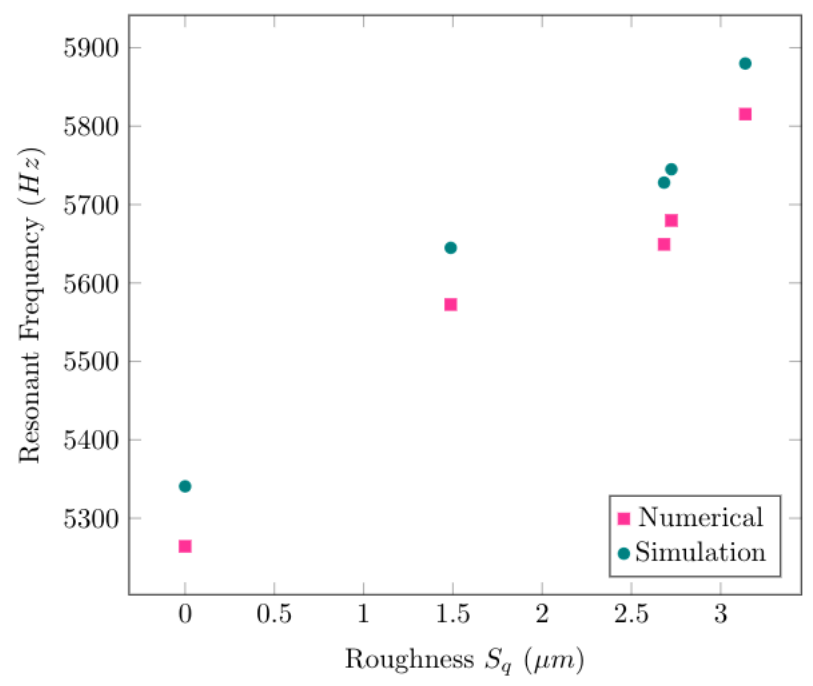

Fig. 7: Surface roughness vs resonant frequency plot of a $100 \mu \mathrm{m}$-thick silicon cantilever for both numerical model calculations and FEM simulations. Data point shows that the resonant frequency increases with surface roughness. The resonant frequency calculated using the numerical model shows about $1 \%$ error from the FEM simulations for all samples.

The first cantilever was set to have no roughness and serves as the ideal sample. For this cantilever, the analytical model gives a natural/resonant frequency of $5815.4 \mathrm{~Hz}$ while FEM simulation gives a resonant frequency of $5879.9 \mathrm{~Hz}$, showing a $1 \%$ difference between the two methods. The other four cantilevers were incorporated with roughness by having the midline of each sample roughness in figure 1 to be exactly at the $100 \mu \mathrm{m}$ height of each beam as illustrated in figure 6 . This was done to ensure each of the four cantilevers will have the same geometric (length, width and height) and material properties. In this way, the change of roughness can be evaluated fairly. The numerical model calculations consistently show around $1 \%$ error compared to FEM simulations. The resonant frequency is also observed to increase along with surface roughness. As the roughness increases, the farther the beam frequency is from its designed resonant frequency. Analysis indicates that an approximately $1.5 \mu \mathrm{m} S_{q}$, roughness, deviates the frequency to $308.31 \mathrm{~Hz}$ from the ideal frequency of the beam with dimensions $5120 \times 1280 \times 100 \mu \mathrm{m}^{3}$.

\section{Conclusion}

A numerical model to determine the natural frequency of a beam with surface roughness was developed. This was done by treating the varying heights across the length of the beam as rectangular regions where moment of inertia is calculated. A simple triple integration of these rectangular region gives the moment of inertia of the beam with roughness. Along with surface roughness, this model also incorporates the device properties of the beam to predict its natural/resonant frequency. The numerical model discussed in this paper can be used to predict surface roughness effects on the resonant frequency of a cantilever beam prior to fabrication. In doing so, adjustments on the design of the MEMS device can be made in order to handle surface roughness effects. Not only that, the numerical model can also be used to determine tolerances with regards to surface roughness, which can be beneficial in designing a device's fabrication process. The numerical model can be further expanded to include surface roughness effects on other energy harvester structures which offers better average strain to increase output power for energy harvesting applications.

\section{Acknowledgments}

The authors would like to give their thanks to Philippine-California Advanced Research Institute - Commission on Higher Education (PCARI-CHED) for the financial support in making this research work possible. 


\section{References}

[1] S. Gopalakrishnan, A. Dasgupta, and D. Nair. "Study of the Effect of Surface Roughness on the Performance of RF MEMS Cpacitive Switches Through 3-D Geometric Modeling". Journal of the Electron Devices Society, 10.1109/JEDS.2016.2582833.

[2] H.L. Xiao, T.T, Z. Tao and H. Li. "Effects of deep reactive ion etching parameters on etching rate and surface morphology in extremely deep silicon etch process with high aspect ratio". Advances in Mechanical Engineering, vol. 9, no. 12, pp. 1-19, 06 2017.W.-K. Chen, Linear Networks and Systems (Book style). Belmont, CA: Wadsworth, 1993, pp. $23-135$.

[3] G. Yuxuan, et. al, "Surface roughness: A review of its measurement at micro-/nano-scale", 2018, January, volume 3, Physical Sciences Reviews, 10.1515/psr-2017-0057.

[4] D. Mondal et. al., "Comsol based multiphysics analysis of surface roughness effects on capacitance in RF MEMS varactors". Proceedings of the COMSOL Conference 2010 India, 2010.

[5] M. Gurgoze. On the eigenfrequencies of a cantilever beam with attached tip mass and a spring-mass system. Journal of Sound and Vibration. No. 190. pp. 149-162. 10.1006/jsvi.1996.0053.

[6] B. Wang, Z. Wang, and X. Zuo. "Frequency Equation of Flexural Vibrating Cantilever Beam Considering the Rotary Inertial Moment of an Attached Mass". Recent Advances in the Application of Differential Equations in Mechanical Engineering Problems. Volume 2017. Article ID 1568019.

[7] Kumar, Vipin \& Singh, Kapil \& Gaurav, Shwetanshu. (2015). Analysis of Natural Frequencies for Cantilever Beam with I-and T-Section Using Ansys. International Research Journal of Engineering and Technology. No. 2. pp. 1013-1020.

[8] A. G. Nejad and J. Y. Hasani. Effects of contact roughness and trapped free space on characteristics of rf-mems capacitive shunt switches. In 2015 IEEE International Conference on Electron Devices and Solid- State Circuits (EDSSC), pages 721-725, June 2015.

[9] R. E. Kalman, "New results in linear filtering and prediction theory," J. BasicEng., vol. 83, no. 4, pp. 95-108, 1961.

[10] R. A. Alam, M.A. Palit, S. "Implications of rough dielectric surfaces on charging-adjusted actuation of RF MEMS. IEEE Electron Device Letter, vol. 35, No. 9, 2014 